\title{
Theorie als Notverordnung
}

\author{
Benötigt die Betriebswirtschaftslehre eine neve Theorie der Unternehmung \\ zur Fundierung von Umweltmanagementsystemen? Diese Frage ist wiederholt \\ Gegenstand der Diskussion. Mit folgendem Beitrag soll deshalb eine lockere \\ Folge von Beiträgen im Informationsdienst eingeleitet werden, die verschiede- \\ ne Theorieangebofe für die Bearbeitung von Fragen der ökologischen Unterneh- \\ mensführung auf ihre Brauchbarkeit und Reichweite abklopfen.
}

$\mathrm{D}$ Von Reinhard Pfriem ieser Beitrag sollte zunächst als Pro-Part einer Kontroverse über die Notwendigkeit einer neuen Theorie der Unternehmung erscheinen. Der für den Contra-Beitrag vorgesehene Kollege sah sich aus Zeitgründen nicht in der Lage, für diese Ausgabe einen Beitrag zu liefern. Trotz manchem Disput miteinander in vergangenen Jahren waren sowohl er als auch ich der Auffassung, daß die Konstruktion eines Gegensatzes zweier Lager in dieser Frage mindestens nicht mehr zeitgemäß und sachgerecht wäre. Insofern ist es bedauerlich, wenn Weber (übrigens mit Bezugnahme auf meinen überarbeiteten Vortrag) in seinem Vorwort zu dem gerade erschienenen Band der Pfingsttagung des Verbandes der Hochschullehrer für Betriebswirtschaftslehre 1996 (Thema: Umweltmanagement) abermals die Behauptung vom für die Diskussion im Umweltmanagement typischen Gegensatz aufstellt - bemerkenswerterweise ohne eine einzige Ausführung dazu, worin denn dieser bestehe. (1)

Nun stecken in der Formulierung der oben genannten Frage mehrere Probleme, die einen sinnvollen Auftakt für die nachfolgenden Überlegungen darstellen. So steht hinter der Frage, ob denn die Betriebswirtschaftslehre eine neue Theorie der Unternehmung brauche, die stillschweigende Annahme, daß sie eine hat diese Annahme ist allerdings vor dem Hintergrund einer dem Gewicht nach paradigmatischen Verschiebung von der Gutenbergschen betriebswirtschaftlichen Theorie hin zu einer angewandten Managementlehre eher fragwürdig. Und die Spezifizierung, ob denn die Betriebswirt- schaftslehre eine neue Theorie der Unternehmung zur Fundierung von Umweltmanagementsystemen brauche, macht wiederum zwei Unterstellungen, gegen die einiges einzuwenden wäre:

- Mindestens ein großer Teil derjenigen, die sich als akademische Betriebswirte mit Umweltmanagementsystemen bzw. allgemein mit Fragen der ökologischen Unternehmensführung auseinandersetzen, scheint das Fundament einer Theorie der Unternehmung dafür nicht für erforderlich $\mathrm{zu}$ halten, einschließlich einer Beschäftigung mit dem, was heute - in großer Mehrheit eher volkswirtschaftlicher oder soziologischer Provenienz - als Unternehmenstheorie existiert. Entweder wird die Sache sowieso "sehr praktisch" (d.h. ohne theoretische Reflektion mit etwas systematischem Anspruch) aufgezogen oder als Spezialisierung aus dem eigenen betriebswirtschaftlichen Funktionsbereich heraus entwickelt.

- Diejenigen, die sich mit der Konzeptionierung oder praktischen Umsetzung von Umweltmanagementsystemen beschäftigen, scheinen dafür in der Regel nichts von dem zu brauchen, was gemeinhin unter Unternehmenstheorie zu verstehen ist. Auf dem Wege zu einer effizienten und kostengünstigen Umsetzung der EGÖko-Audit-Verordnung in einem speziellen Unternehmen würde man auf den ersten Blick wohl eher vermuten, daß sie stört. Die Marktverhältnisse bei der Zertifizierung, speziell der Mechanismus, daß für das bloße Zertifikat weniger kritische externe Gutachter die bequemeren sind, die formal strenge Vorgabe der Vorgehensweise der Verordnung selbst - alles das schreit nicht nach Theorie.

\section{Liebe auf den zweiten Blick}

Liebe zur Theorie der Unternehmung als möglichem Fundament für Umweltmanagementsysteme kann allenfalls gelingen als Liebe auf den zweiten Blick. Was freilich wäre die Liebe auf den ersten Blick ohne jene auf den zweiten Und tatsächlich gerät şchon ein aufmerksamer Beobachter der EG-Öko-Audit-Verordnung ins Stutzen, wenn er auf die Verpflichtung des Unternehmens stößt, im Vorfeld einer solchen Zertifizierung eine Umweltpolitik ausarbeiten sowie Umweltziele und ein Umweltprogramm ausarbeiten zu müssen. Für den betrieblichen Alltag eines kleinen und mittelständischen Unternehmens scheint dies zunächst eine zu hohe und eher abwegige Anforderung zu sein, und allzu pragmatische Berater verstärken sehr gern solche Skepsis und liquidieren damit sämtliche betriebswirtschaftlichen Möglichkeiten, die in diesen Anforderungen stecken.

Der zweite Blick könnte nun die Augen dafür öffnen, daß keineswegs nur für große, sondern auch für kleine und mittelständische Unternehmen die Besinnung auf die eigenen Wünsche, Ziele und Fähigkeiten, damit die Entbergung der eigenen strategischen Erfolgspotentiale völlig neue Wege in die Unternehmenszukunft öffnen kann. Es geht also um unternehmenspolitische Selbstbeobachtungen und Selbstbeschreibungen(2), über die normative Grundlagen der Unternehmensführung nach innen und außen gewonnen werden können. Mit anderen Worten: diese müssen passen, $d$. h. mit der gegebenen bzw. gewinnbaren Unternehmensphilosophie, -kultur oder -ethik übereinstimmen. Die positive Norm der Entwicklung von Unternehmensleitbildern hat Ortmann übrigens sehr schön ausgedrückt, wenn er mit literarischem Bezug auf Kleist „von deren allmählicher Verfertigung im Handeln" spricht, oder - mehr mit Giddens als mit Kleist - von ,rekursivem Schleifen sozialer Praxis". (3)

Unter wesentlicher Bezugnahme auf Giddens setzen sich Ortmann, Sydow und Windeler auch in dem soeben erschienenen Band über Theorien der Organisation 
(4) gründlich mit der Rekursivität menschlichen Handelns auseinander, „die darin liegt, daß wir handelnd genau diejenigen Strukturen als Resultat hervorbringen, die sodann unser weiteres Handeln ermöglichen und restringieren." Hier wird von organisationstheoretischbetriebswirtschaftlicher Seite darauf hingewiesen, daß Strukturen eben nicht nur restringieren, sondern auch ermöglichen. Stellungnahmen von Arbeitgeberverbänden und -verbandsfunktionären lehren einen das in der Regel nicht gerade, und passend dazu ist auch die vorherrschende ökonomische Theorie gebaut. Für den main-stream der ökonomischen Theorie (für die nach gängiger fragwürdiger Arbeitsteilung die Volkswirte zuständig sind) heißt es mit Kirchgässner "Verhalten von Individuen wird erklärt, indem unterstellt wird, daß sie rational handeln."(5) (Dabei ist es übrigens dem bedauerlicherweise in den main-stream-economics ebenfalls noch vorherrschenden methodologischen Individualismus zu ,verdanken“, daß die ehernen Sätze in der Regel eher auf die Beziehung Individuum zu Struktur rekurrieren, als auf jene von Organisation (etwa Unternehmung) zu (sie umgebender) Struktur.)

Das, was ich seit einiger Zeit als ökonomisches Verhaltensmodell bezeichne, zieht sich leider sehr penetrant durch die Geschichte der modernen Wirtschaftstheorien. Als vornehmste Aufgabe der ökonomischen Akteure wird weniger gesehen, ihre tatsächlich vorhandenen Freiräume sinn- und verantwortungsvoll wahrzunehmen, sondern vielmehr sich an externe Veränderungen möglichst rasch und effizient anzupassen. Der theoretische Ökonom dieses Schlages hat nicht nur ein ernüchtertes, sondern ein recht trostloses Menschenbild, wenn er meint, Veränderungen im menschlichen Verhalten über Veränderungen der Restriktionen erklären zu müssen.(6) Aber diese Grundorientierung prägt die ökonomische Theorie des zwanzigsten Jahrhunderts: so definiert etwa Hayek ganz prinzipiell den Charakter der ökonomischen Probleme(7), und auch für Barnard hieß die entscheidende Aufgabe von Unternehmensorganisationen Anpassung. (8)
Für mich ist der Befund, daß so zahlreiche Praktiker und Theoretiker an der reaktiven und anpassungsorientierten Sichtweise festhalten, schon seit längerem ein bemerkenswertes Phänomen, hat es doch auf beiden Ebenen eine recht starke Selbstamputation zur Folge: da jammert der Präsident des DIHT (wie jener des BDI) ständig über die zu hohen Kosten am Standort Deutschland, statt sich in kreativen Ideen und Perspektiven einer innovativen und zukunftsorientierten Wirtschaft zu ergehen; und auch der dazu passende Theoretiker bringt sich um jedwede Chancen, über das Anpassungslernen hinaus das Veränderungslernen und das Lernen zu lernen aufgeschlossen untersuchen zu können. (9)

Was bleibt, kann in dem hier vorgegebenen Rahmen nur völlig grob und knapp angedeutet werden. Volkswirte und Soziologen sollten sich, soweit es sie betrifft, aus der wohl fachbedingten Verstrickung befreien, einseitig $\mathrm{zu}$ viel Gewicht auf die Rahmenbedingungen zu legen, d.h. auf Strukturen als Restringierendes und überhaupt nicht Ermöglichendes. Die Betriebswirtschaftslehre sollte sich über die merkwürdige Haltung hinausbewegen, einen die Handlungsbedingungen nicht reflektierenden Instrumentalismus als Kerngeschäft zu betreiben und an Sonntagen, an denen Theorie angesagt ist, schamhaft, fast sklavisch auf die Unternehmenstheorien volkswirtschaftlicher Provenienz zu schielen.

Neben der schon angedeuteten organisationstheoretischen Öffnung (die allerdings stärker, expliziter und präziser als bisher das Agieren von Unternehmensorganisationen in gesellschaftlichen Umfeldern als ihren Bearbeitungsgegenstand definieren müßte von Gesellschaft als konkreter, gesellschaftlichen Veränderungen und Entwicklungsmöglichkeiten ist trotz des Untertitels in dem Band von Ortmann, Sydow und Türk kaum die Rede) gibt es einige begonnene Diskurse in der betriebswirtschaftlichen Fachdisziplin, die zu Hoffnung Anlaß geben:

- die Auseinandersetzung mit selbstorganisatorischen Prozessen (10) sowie den Bedingungen und Möglichkeiten organisationalen Lernens,
- das Ringen um eine „Integrative Wirtschaftsethik"(11), die zuerst analytisch (hinsichtlich der normativen Aufgeladenheit jeglichen Wirtschaftens) und darauf aufbauend ethisch-normativ der „Transformation der ökonomischen Vernunft"(12) beizukommen versucht (bei Peter Ulrich wie bei mir irrt übrigens Ortmann(13), wenn er das illustrative Bild ökologisch-ökonomischer Schnittmengen als wesentliche theoretische Erklärung für das Gelingenkönnen ökologischer Unternehmensführung unterstellt),

- neuere Entwicklungen in der Theorie strategischer Unternehmensführung, wo die intensivere Diskussion der ResourceDependence-Theorie und des Resourcebased View zu gründlicherer theoretischer Beschäftigung mit den endogenen Potentialen von Unternehmen führt,(14)

- die Überwindung einseitig absatzorientierten Marketings durch Analyse von Produktkulturen (15), v. a. die Überwindung von Marketing als solchem durch Kommunikation (16)

- das Bemühen, aus der ökologischen Erweiterung einer auf das Gutenbergsche Paradigma der Optimierung als intern vorgestellter betrieblicher Leistungserstellungsprozesse bezogenen Konzeption zur Infragestellung exakt definierter Schnittstellen zwischen Produktion und Konsumtion zu gelangen (17).

Das alles sind Ansätze, wo manche Erweiterungen vorstellbar sind. (18) Nicht der unwichtigste Hinweis lautet, daß alles hier Ausgeführte bei näherem Hinsehen (dem wievielten Blick?) natürlich nicht nur auf das ökologische Handlungsfeld von Unternehmen zu beziehen wäre, sondern auch auf alle übrigen. Aber das wäre eine andere Geschichte ...

\section{Anmerkungen}

1) Vgl. Weber,J. (Hg.): Umweltmanagement, Aspekte einer umweltbezogenen Unternehmensführung, Stuttgart 1997, Vorwort $0 . S$.

2) Im systemtheoretischen Kontext finden sich dazu einige interessante Überlegungen bei Kasper,H.: Die Handhabung des Neuen in organisierten Sozialsystemen, Berlin u.a. 1990; vgl auch ders.: Neuerungen durch selbstorganisierende Prozesse, in: Staehle,W.H./Sydow,J.(Hg.): Managementforschung Bd. 1, Berlin-New York 1991.

3) S. Orrmann,G.: Das Kleist-Theorem. Über Ökologie, 
Organisation und Rekursivität, S.57 in: (Hg.) Birke,M./ Burschel,C./Schwarz,M.: Handbuch Umweltschutz und Organisation, München/Wien 1997.

4) Ortmann,G./Sydow,J./Türk,K.(Hg.): Theorien der Organisation. Die Rückkehr der Gesellschaft,Opladen 1997.

5) Kirchgässner,G.: Homo oeconomicus: das ökonomische Modell individuellen Verhaltens und seine Anwendung in den Wirtschafts- und Sozialwissenschaften, Tübingen 1991, S.18.

6) So aber Kirchgässner a.a.0. S.39.

7) Vgl. von Hayek, F.A.: The Use of Knowledge in Society, in: American Economic Review, vol. 35 (1945), S.519530.

8) „.....weshalb wir unser Hauptaugenmerk auf diejenigen Prozesse richten werden, durch die...diese Anpassung erreicht wird." (Barnard, Ch.: The functions of the executive, Cambridge/Mass. 1938, S.6).

9) Vgl: dazu aufbauend auf Bateson Argyris, Ch./Schön, D.A.: Organizational Learning, Reading, Mass. 1978.

10) Ein Pionier in unserem Fach dazu ist Probst,G.J.B.: Selbst-Organisation - Ordnungsprozesse in Organisationen aus ganzheitlicher Sicht, Berlin/Hamburg 1987. Zu Schwächen dieses Zugriffs vgl. Pfriem,R.: Unternehmenspolitik in sozialökol. Perspektiven, 2.A. Marburg 1996, S.154 f.

11) So der Titel des soeben erschienenen neven Werkes von Peter Ulrich, Bern/Stuttgart 1997.

12) So der Titel vonPeter Ulrichs grundlegendem Werk, Bern/Stuttgart 1986.

13) Ortmann, G.: Das Kleist-Theorem, a.a.0. S.59.

14) Vgl. dazu zu Knyphausen-AufseB,D.: Auf dem Weg zu einem ressourcenorientierten Paradigma? In: ( $\mathrm{Hg}$.) Ortmann,G./Sydow,J./Türk,K. a.a:0. S.452 ff.

15) Neben dem hier wichtigen soziologischen Klassiker von Schulze,G.: Die Erlebnisgesellschaft: Kultursoziologie der Gegenwart, Frankfurt/New York 1993 sei verwiesen auf den instruktiven Sammelband von Eisendle, R./Miklautz ,E.(Hg.): Produktkulturen: Dynamik und Bedeutungswandel des Konsums, Frankfurt/New York 1992.

16) S. dazu Zerfaß,A.: Unternehmensführung und Öf-fentlichkeitsarbeit. Grundlegung einer Theorie der Unternehmenskommunikation und Public Relation, Opladen 1996.

17) Ein erster, allerdings noch nicht weiterverfolgter Ansatz der betriebswirtschaftlichen Produktionstheorie hierfür fand sich bei Dyckhoff.

18) Die der Unternehmung als strukturpolitischem Akteur verfolgt mit besonderer Konsequenz Uwe Schneidewind, s. zuletzt ders.: Ökologische Reorganisation von Branchen. Von der Mikropolitik in der Organisation zur Strukturpolitik der Organisation, in: Birke/Burschel/Schwarz 1997 (s.o.), $226 \mathrm{ff}$.

\section{Der Autor}

Dr. Reinhard Pfriem ist Professor für Allgemeine Betriebswirtschaftslehre, Unternehmensführung und betriebliche Umweltpolitik an der Carl von Ossietzky Universität Oldenburg.

Kontakt: Birkenweg 5, Postfach 2503, 26111

Oldenburg, Tel. 0441/798-8345/8356, Fax -8341

Integrierte Managementsysteme für kleine und mittlere Unternehmen

\title{
Einheitsbrei oder Menü in drei Gängen
}

\author{
Mit der Einführung never Gesetze, Normen und Verordnungen in den Bereichen \\ Qualitätsmanagement, Arbeitsschutz und Umweltschutz stellt sich immer \\ stärker die Frage nach der Installation integrierter Managementsysteme. \\ Aber wie könnte ein solches Systemm aussehen? Das Instifut für ökologische \\ Wirtschaftsforschung hat zur Konzeption und Einführung derartiger Manage- \\ mentsysteme einen Vorschlag erarbeitet.
}

$\mathrm{D}$ Von Michael Steinfeldt ie Dynamik bei der Erteilung von Zertifikaten für Qualitätsmanagementsysteme nach DIN ISO 9000 ff. in Deutschland hält bis heute an. Waren Ende 1992 erst circa 700 Unternehmen zertifiziert, waren es im September 1995 bereits 7000 . Mit der EG-ÖkoAudit-Verordnung (EMAS) sowie der DIN ISO 14000 begann zeitversetzt Ende 1995 eine ähnliche Entwicklung zu validierten/zertifizierten Umweltmanagementsystemen, von denen es Mitte 1997 über 700 in Deutschland gibt.

$\mathrm{Zu}$ Managementsystemen für Arbeitssicherheit wird es in näherer Zukunft zwar keine ISO-Norm geben, aber das seit August 1996 in Kraft getretene neue Arbeitsschutzgesetz wird weitreichende Auswirkungen hin zu einem ganzheitlichen, systematischen Arbeitsschutz haben. Speziell für die Lebensmittelbranche steht außerdem im Zuge der Umsetzung der Verordnung für Lebensmittelhygiene (die Verordnung tritt im Februar 1998 in Kraft) die Forderung nach Einführung eines Managementsystems in dem Bereich Hygiene (HACCP) im Raume.

Mit diesen neuen Gesetzen, Normen und Verordnungen stellt sich immer stärker die Frage nach integrierten Managementsystemen, die diese verschiedenen Aspekte berücksichtigen und zusammenführen. Doch zuvor einige Bemerkungen zum Stand in den einzelnen obengenannten Bereichen.

\section{Bisherige betriebliche Praxis}

Betrachtet man die Erfahrungen bei der Einführung von Systemen des Qualitätsmanagements, dann muß kritisch festgehalten werden, daß in vielen kleinen und mittleren Unternehmen (KMU) sehr statische Systeme eingeführt wurden, die sehr schematisch die 20 Elemente der Norm DIN EN ISO $9000 \mathrm{ff}$ abarbeiten, aber die originären betrieblichen Abläufe nur unzureichend abbilden. Dies führte zu gewissen Vorurteilen gegenüber Normierungen allgemein, vor allem was Bürokratie, Formalismus und das Zementieren schlechter Abläufe betrifft. Die Berücksichtigung des Umweltschutzes wird aus einer derartigen Sichtweise heraus oft nur in der Ergänzung eines „21. Kapitel Umweltschutz“ des bestehenden Systems und in der Einfügung des Begriffes Umwelt in den anderen Kapiteln gesehen.

Den Anforderungen des Umweltschutzes trägt die EMAS in anspruchsvoller Art und Weise Rechnung, wobei sich aber in kleinen Unternehmen oft die Frage stellt, ob aufgrund der geringen Relevanz der betrieblichen Umweltauswirkungen und den notwendigen Personalkapazitäten die Einführung eines eigenständigen Umweltmanagementsystems überhaupt gerechtfertigt erscheint.

Der betriebliche Arbeits- und Gesundheitsschutz ist ein Feld mit entwickelten Beziehungen zwischen den Akteuren und Gremien. Die Akteure des Arbeitsschutzes verfügen meist über jahrelange 
(c) 20I0 Authors; licensee IÖW and oekom verlag. This is an article distributed under the terms of the Creative Commons Attribution Non-Commercial No Derivates License (http://creativecommons.org/licenses/by-nc-nd/3.o/), which permits unrestricted use, distribution, and reproduction in any medium, provided the original work is properly cited. 